\title{
THE STUDY IN USING AN AUTONOMOUS ROBOT FOR PAVEMENT INSPECTION
}

\author{
Jia-Ruey Chang \\ Associate Professor \\ Department of Civil Engineering \\ MingHsin University of Science \& Technology \\ No. 1, Hsin-Hsing Road, Hsin-Chu, 304, \\ Taiwan \\ jrchang@must.edu.tw \\ Shih-Chung Kang \\ Assistant Professor \\ Department of Civil Engineering \\ National Taiwan University \\ No. 1, Sec. 4, Roosevelt Road, Taipei, 106, \\ Taiwan \\ sckang@caece.net
}

\author{
Yuan-Hsu Tseng \\ Graduate Student \\ Department of Civil Engineering \\ National Taiwan University \\ No. 1, Sec. 4, Roosevelt Road, Taipei, 106, \\ Taiwan \\ b92501045@ntu.edu.tw

\section{Chih-Hwang Tseng} \\ Director \\ Division of Transportation Engineering \\ Institute of Transportation \\ Ministry of Transportation and Communications \\ No. 240, Tun-Hwa North Road, Taipei, 105, \\ Taiwan \\ Tseng@iot.gov.tw
}

\author{
Po-Huei Wu \\ Associate Researcher \\ Division of Transportation Engineering \\ Institute of Transportation \\ Ministry of Transportation and Communications \\ No. 240, Tun-Hwa North Road, Taipei, 105, Taiwan \\ witch@iot.gov.tw
}

\begin{abstract}
Traditionally pavement inspections are conducted using manually operated instruments, and hence are very labor-intensive. In this study, the authors propose the idea of using an autonomous robot to perform the pavement inspections, including for evenness and distress. Because inspection instruments are assumed to be mounted on the robot's platform, this robot is capable of inspecting pavement conditions while at the same time planning the upcoming motion path according to the inspection results. Working concurrently in this way significantly reduces the overall inspection time and amount of data to be stored. The authors propose four motion planning methods for robots: a transversal path planning method, a longitudinal path planning method, a random walk method, and an obstacle avoidance method. This paper presents the application of the motion planning methods to different pavement inspection tasks. It also illustrates the inspection process in a predefined inspection area, and in an inspection area that has random obstacles. The results of this research are suitable for use with a commercial robot to achieve the goal of robotic pavement inspections.
\end{abstract}

\section{KEYWORDS}

Autonomous Robot, Pavement Inspection, Pavement Evenness, Pavement Distress, Path Planning 


\section{INTRODUCTION}

Among common infrastructure, roads are one of the most difficult to maintain. The road usually locates broadly in various weather and traffic conditions. They are often worn due to traffic passage, changeful temperatures and the random impacts of vehicles. Different deterioration rates and randomly occurring distresses increase the complexity of maintaining and managing the pavements on roads.

Since road users can easily perceive the pavement problems, most governments try to maintain the pavements as best they can. In most cases, road authorities periodically inspect pavements and keep the conditions above an acceptable serviceability (the quality of pavement for road users) and determine appropriate and timely maintenance and rehabilitation (M\&R) activities. Appropriate $\mathrm{M} \& \mathrm{R}$ activities not only prolong pavement life but also save life-cycle costs; as well as provide comfortable and safer pavements for road users [1].

Successful M\&R activities rely on effective pavement inspections with proper approaches and within budget. It also requires careful allocation of limited resources such as inspection instruments and operators. However, in current inspection processes, the inspection plans are developed subjectively by engineers prior to inspections. After planning is complete, the inspection tasks are conducted using manually operated instruments. These inspections usually include repetitive and tedious procedures, and often require a great amount of time for post-analysis.

In the study, the authors pioneered the idea of using an autonomous robot to perform the pavement inspections to reduce the overall resource cost. With self-controlled motion and automatic path planning, robots can reduce the variation and uncertainty of inspections; and, the reliability of inspections would be improved.

\section{PAVEMENT INSPECTION}

The serviceability provided by pavement is highly related to surface condition. In general, the surface conditions are monitored by periodically collecting the longitudinal evenness (roughness), transversal evenness (rutting), and distress information using of manifold commercial equipment [2].

\subsection{Pavement Evenness}

For flexible pavement, roughness and rutting are the variations of longitudinal and transversal profiles respectively. Both are important for comfortable driving or riding for road users. In the past decades, the instruments developed for roughness inspection were improved through automation. In general, the roughness instruments are grouped into four types: Profiler/Profilograph, Response-Type Road Roughness Measuring Systems (RTRRMSs), Inertia Profilometer/Lightweight Profilometer, and Walking Profiler [2].

\subsection{Pavement Distress}

A pavement distress survey is conducted to monitor the present pavement condition and determine the appropriate M\&R activities. The common distress types include alligator cracking, longitudinal cracking, transverse cracking, block cracking, rutting, and patching. Toward the end of the 1970s, Darter and Shahin [3] developed the Pavement Condition Index (PCI); which, has been referred as ASTM D6433 [4] for roads and parking Lots and ASTM D5340 [5] for airfield pavement. Manual and automatic approaches are the two major ways, adopted all over the world, to conduct a pavement distress survey. The manual approach is conducted through visual observation. The severity and coverage of each distress on one pavement unit are identified and recorded using pen and paper or a personal digital assistant (PDA). The PCIs can be computed immediately using the PDA or manually during the post-processing. The automatic approach is to first capture pavement images, and then the severity and coverage of each distress are automatically identified through image processing algorithms. The PCIs and their accuracy are computed immediately or semi-automatically during the post-processing. However, present image processing merely focuses on linear cracking and potholes thus could not replace the manual approach. Significant developments have taken place as far as automating the distress measurement is concerned. There are lots of inertial profilers that automate the 
complete distress date collection. More literatures can be referred as $[6,7]$.

Most evenness and distress inspections on broad pavements are conducted using a repetitive and time-consuming procedure that uses human-operated instruments. Because the inspectors know little about the inspection results at the time of the inspection, data collected often includes a significant amount of redundant points. To solve this problem, this study proposes the idea of applying autonomous robots to pavement inspections by utilizing the robot's features of mobility, high accuracy, and time and labor efficiencies. The robot will analyze the inspection data in real time, and plan the upcoming motion immediately. This helps us collect pavement data in a more effective way.

\section{AUTONOMOUS ROBOT}

An autonomous robot is an intelligent mechanical creature that can function autonomously. "Intelligent" implies that the robot does not do things in a mindless, repetitive way. The "mechanical creature" means that our scientific technology uses mechanical building blocks, not real biological components. It also emphasizes that a robot is not the same as a computer. A robot may have a computer as a building block, equivalent to a nervous system or brain, but the robot is able to interact with its world: move around in it and manipulate it. A computer does not move around of its own accord. "Function autonomously" indicates that the robot can operate, self-contained, under all reasonable conditions without requiring recourse to a human operator. Autonomy means that a robot can adapt to changes in its environment (the lights get turned off) or itself (a part breaks) and continue to reach its goal [8].

The general framework for a pavement inspection system is configured as shown in Figure 1(a). The conveyer (such as a vehicle) carries various inspection instruments and is controlled by an inspector in order to collect vast amount of data. Both longitudinal evenness (roughness) and transversal evenness (rutting) can be automatically and immediately analyzed during inspections. But, PCI computation has to be done at post-processing and relies on a computer. PCI computation takes the same $(1: 1)$ or more time than inspection. During inspections, inspectors are usually unable to know the results of the data being collected. They cannot adjust the inspection paths according to the inspection results in real-time. To avoid losing any important data points, the strategy they usually use is to collect all data points over all of the inspection area.

An autonomous robot consists of three parts, as shown in Figure 1(b): (1) a sensor unit; (2) an actuator unit; and (3) a logic unit [9]. The sensor unit works using instruments, such as an ultrasonic [10]/ laser range finder [11], image capture equipment [12], and an odometer in the pavement inspection system. The actuator unit [13] is responsible for robot motions and works as a conveyer in the pavement inspection system. The logic unit has computational power, which is similar to data analyses modules in the pavement inspection system. The logic unit is able to perform real-time computation and decision-making.

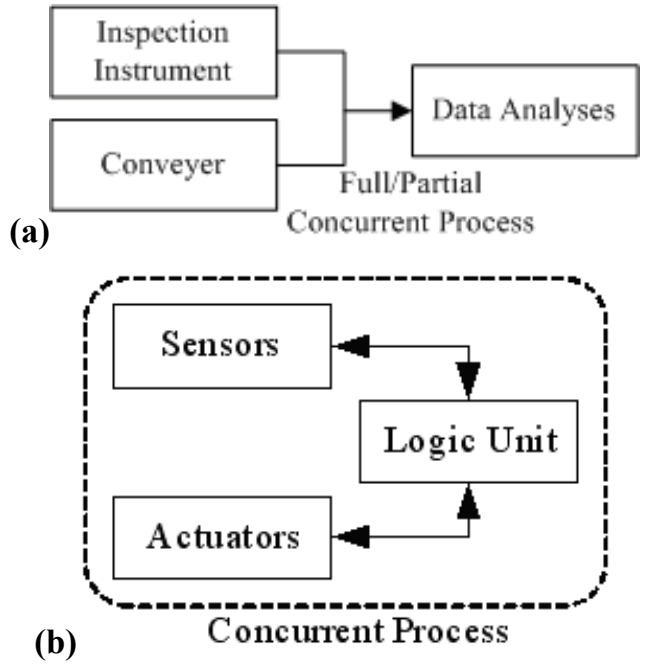

Figure 1 The Systematic Framework: (a) Present Pavement Inspection System; (b) Autonomous Robot

One autonomous robot framework for pavement inspections is proposed as in Figure 2. The sensor unit of the robot is combined with pavement inspection instruments and mounted on the robotic platform. By integrating the results from the logic unit of the robot and pavement data analyses, the robot is capable of intelligently planning the coming behaviors. Since the sensing and computing are synchronous, the time required for the processes of data collection and analyses can 
be reduced significantly. The concurrent process also can reduce the data storage requirements.

\section{ROBOTIC INSPECTION}

Motion planning is one of the most important issues in developing a robot for pavement inspections. The major goal of the pavement inspections is to survey the overall pavement conditions and at the same time probe the pavement problems.

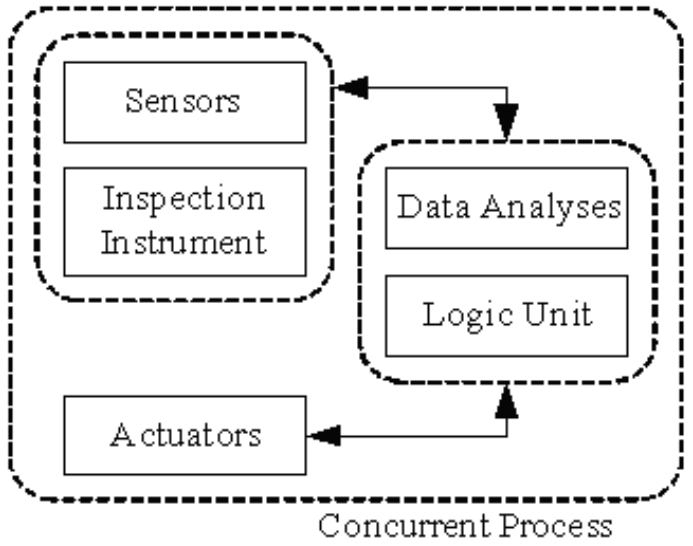

Figure 2 The Autonomous Robot Framework for Pavement Inspections

A typical scenario for pavement inspection is as follows. Given a predefined inspection area, the inspector has to run over this area at a certain speed. This speed is sufficient for the inspection instrument to obtain the information correctly. In the case of manual roughness inspection, for example, the inspector has to haul the walking profiler (a roughness inspection machine) along both wheel paths within the speed limit $(<800$ $\mathrm{m} / \mathrm{hr}$ ). To plan the motion of the robot for the purpose of pavement inspection, we have to develop methods to define inspection paths. We also need to develop a method to ensure that the robot avoids all possible obstacles, including both static obstacles and dynamic obstacles. Additionally, a method for random inspection that enables the robot to run over the inspection area using a random procedure is also necessary if we would like to introduce intelligent sampling strategies for reducing the inspection time and the data storage requirements.

\subsection{Motion Planning Methods}

The authors proposed four motion planning methods: a transversal path planning method; a longitudinal path planning method; a random walk method; and an obstacle avoidance method, to enable robots to conduct pavement inspections autonomously. They are explained as follows:

- The transversal path planning method is to plan inspection paths that are perpendicular to the roadway. Because we assume the inspection area is given before the start of inspection, we can use a computer program to recognize the road boundary and generate the lines perpendicular to the boundary. By linking the lines, we are able to obtain a transversal inspection path.

- The longitudinal path planning method is to plan paths that are parallel to the roadway. Similar to the processes used in the transversal path planning method, we can generate the lines parallel to the roadway and find the longitudinal path by linking these parallel lines.

- The random walk method is to plan random movement for the robot. The basic technique is to assign random targets for a robot and ask it to move toward the targets consecutively.

- The obstacle avoidance method is a function that helps the robot avoids the obstacles in the physical environment. This method is achieved by continuously retrieving information from the sensors. When an obstacle is found, the robot will turn toward in the direction opposite to the robot's motion. In some cases, backward movement and random movement are also involved to avoid obstacles.

\subsection{Robotic Pavement Inspection Paths}

In this research, the authors proposed a method for robots to conduct pavement inspections. This method integrates the four aforementioned motion planning methods. We first target the scenario in which a robot conducts pavement inspection in a predefined environment with no obstacles. The only constraint for the robot's motion is the boundary of the inspection area. Then, we target a more complicated scenario, an inspection 
environment that has random obstacles, which is closer to what the robot will face in the environments of actual pavement inspections.

Figure 3 shows four possible inspection paths for the first scenario: pavement inspection in a predefined and obstacle-free environment. Figure 3(a) illustrates the inspection path in a transversal mode. For the inspection task related to the transversal direction of a road, such as rutting (the pavement depression along wheel paths caused by tires), the robot may travel along the transversal path as shown in Figure 3(a). These paths can be generated by introducing the transversal path planning method. Figure 3(b) illustrates the longitudinal inspection mode. This mode is ideal for the inspections related to the pavement conditions along the roadway. Roughness inspection and distress survey are two such tasks. This path can be generated by introducing the longitudinal path planning method. Figure 3(c) illustrates a random inspection mode. The random walk motion planning method will facilitate the robot to conduct the random inspection when a sampling inspection is required. Figure 3(d) illustrates the grid mode for inspections. The robot either moves transversely or longitudinally. This mode can be an ideal solution for simultaneous inspections under the condition of roadway with irregular boundary. To generate the path, we can integrate both the transversal path planning method and the longitudinal path planning method.

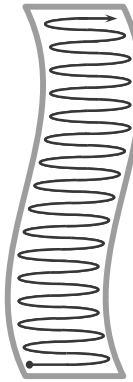

(a) ...

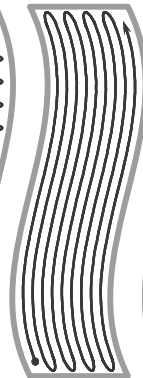

(b)

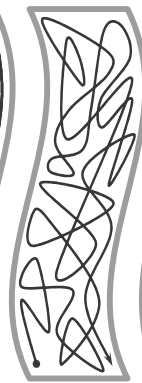

(c)

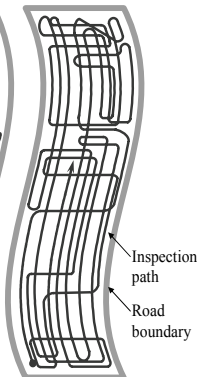

(d)
Figure 3 Pavement Inspection Paths Without

Obstacles: (a) Transversal Mode;

(b) Longitudinal Mode; (c) Random Mode; (d) Grid Mode

Figure 4 shows the scenario in which a robot conducts the inspection in an environment with randomly occurring obstacles. Figure 4(a) illustrates the transversal inspection in an environment with obstacles. By executing the path planning and obstacle avoidance methods simultaneously, we can see that the robot will follow the transversal inspection path in the region without obstacles. While the robot senses an obstacle, it will avoid the obstacle by slightly altering the path. After the obstacle region has been passed, the robot will continue following the predefined transversal path. Figures 4(b), (c) and (d) illustrate the simultaneous execution of the obstacle avoidance method and the longitudinal path planning method, the random walk method, and the combination of transversal and longitudinal path planning methods respectively.
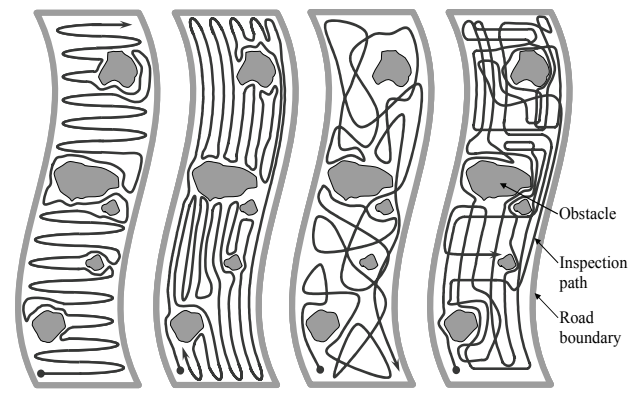

Figure 4 Pavement Inspection Paths With Obstacles: (a) Transversal Mode;

\section{(b) Longitudinal Mode; (c) Random Mode; (d) Grid Mode}

\section{SUMMARY AND FUTURE WORK}

In this paper, we provided a method of using an autonomous robot to perform, what are for people, tedious pavement inspections. A framework that integrates the inspection instruments and the robot was proposed. The robot acts as an intelligent conveyer that carries the inspection instruments to conduct pavement inspections. We also proposed four robotic motion planning methods. They are the transversal path planning method, the longitudinal path planning method, the random walk method and the obstacle avoidance method. We illustrated how to integrate these motion planning methods to facilitate navigating the robot in both an obstacle-free environment and in an environment with random obstacles.

In the future, the authors plan to test the proposed methods in a simulation environment by using virtual reality technologies, such as Microsoft 
Robotics Studio (MRS), to verify the feasibility of these methods applied to pavement inspections. If the virtual robot performs smoothly in the simulation environment, we will link the program to a real robot to extend our work into reality. Additionally, authors will provide some relative performance parameters in the use of robot when compared with a traditional pavement inspection method. The ultimate goal of this research is to develop a robot that is capable of conducting pavement inspections autonomously in an economical and intelligent way.

\section{ACKNOWLEDGMENTS}

The authors would like to thank the financial support provided by the Technology Development Program for Academia, the Ministry of Economic Affairs, Taiwan, under the project of "The Development of Integrated Intelligent Robotics System" (contract no: 95-EC-17-A-04-S1-054).

\section{REFERENCES}

[1] Abaza, K.A., Ashur, S.A., Al-Khatib, I.A. (2004) Integrated Pavement Management System with a Markovian Prediction Model, Journal of Transportation Engineering, Vol. 130, No. 1, 24-33.

[2] Shahin, M.Y. (2005) Pavement Management for Airports, Roads, and Parking Lots, Second Edition, Springer.

[3] Darter, M.I. \& Shahin, M.Y. (1980) Pavement Rehabilitation: Identifying the Need, Journal of Transportation Engineering, Vol. 106, No. 1, $1-10$.

[4] ASTM D6433 (2003) Standard Practice of Roads and Parking Lots Pavement Condition Index Surveys.

[5] ASTM D5340 (2004) Standard Test Method for Airport Pavement Condition Index Surveys.

[6] Wang, K.C.P. (2003) Transportation Research Circular: Automated Imaging Technologies for Pavement Distress Survey, Committee A2B06, Transportation Research Board, National Research Council, Washington, D.C.

[7] Wang, K.C.P. \& Li, X. (1999) Use of Digital Camera for Pavement Surface Distress Survey, Transportation Research Record 1675, Transportation Research Board, National Research Council, Washington, D.C., 91-97.

[8] Murphy, R. (2000) Introduction to AI Robotics, MIT Press.

[9] Kilner, M.J., Alonso-Alonso, M., Fisher, R., \& Lemon, N.R. (2002). Modulation of Synchrony Between Single Motor Units During Precision Grip Tasks in Humans, The Journal of Physiology, Vol. 541, No. 3, 937-948.

[10] Tanzawa, T., Kiyohiro, N., Kotani, S., \& Mori, H. (1995) The Ultrasonic Range Finder for Outdoor Mobile Robots, Proceedings of the IEEE/RSJ International Conference on Intelligent Robots and Systems, Vol. 3, Pittsburgh, USA, August 5-9, 368-373.

[11]Rioux, M. (1984) Laser Range Finder Based on Synchronized Scanners, Applied Optics, Vol. 23, 3837-3844.

[12]Narabe, T., Nara, K., \& Yanagihara, M. (1999) Alignment Device and Method Based on Imaging Characteristics of The Image Pickup System, Retrieved May 25, 2007, from http://www.freepatentsonline.com/5978094.ht $\mathrm{ml}$

[13]Arimoto, S. (1990) Learning Control Theory for Robotic Motion, International Journal of Adaptive Control and Signal Processing, Vol. 4, No. 6, 543-564. 